zwischen ihren Akteuren mit denen der politischen Bildung und ihrer Didaktik beitragen. Wie gut sich entwicklungsbezogene Bildungsarbeit und politische Bildung ergänzen und gegenseitig befruchten können, zeigt die gemeinsame Publikation der Bildungszentrale für politische Bildung von Wolfgang Sander und Annette Scheunpllug „Politische Bildung in der Weltgesellschaft. Herausforderungen, Positionen, Kontroversen" (2011).

\section{Literatur}

Asbrand, B. \& Schröder, U. B. (2008). Evaluation zwischen Praxis und Wissenschaft. Zum Potential der dokumentarischen Evaluationsforschung bei der Evaluation von Schulprojekten und im Feld Globales Lernen. ZEP - Zeitschrift für internationale Bildungsforschung und Entwicklungspädagogik, 31(2), 8-14.

Bergmüller, C., Causemann, B., Höck, S., Krier, J. \& Quiring, E. (2019). Wirkungsorientierung in der entwicklungspolitischen Inlandsarbeit. Münster: Waxmann.

Bergmüller, C., \& Paasch, D. (2008). Evaluation von Lerneffekten in Maßnahmen entwicklungsbezogener Bildungsarbeit. Potentiale und Grenzen. ZEP-Zeitschrift für internationale Bildungsforschung und Entwicklungspädagogik, 31(2), 15-19.

Bergmüller, C., Scheunpflug, A., Franz, J. \& Krogull, S. (2013). Zur Überprüfung entwicklungsbezogenen Lernens. Anmerkungen zum VENRO-Diskussionspapier „Wirkungsorientierung in der entwicklungspolitischen Inlandsarbeit“. Zeitschrift für Evaluation, 12(1), 151-161.
Bergmüller-Hauptmann, C. \& Scheunpflug, A. (2017). Die Evaluation entwicklungsbezogener Bildungsarbeit. In G. Lang-Wojtasik \& U. Klemm (Hrsg.). Handlexikon Globales Lernen. Münster et al.: Klemm \& Oelschläger, 76-81.

Maturana R. \& HumbertoVarela G. F. (1987): Der Baum der Erkenntnis: die biologischen Wurzeln des menschlichen Erkennens. München: Scherz.

Sander, W. \& Scheunpllug, A. (Hrsg.) (2011). Politische Bildung in der Weltgesellschaft. Herausforderungen, Positionen, Kontroversen. Bonn: Bundeszentrale für politische Bildung.

Scheunpflug, A. \& Bergmüller, C. (2014): Kommentar in: VENRO: Wirkungserfassung in der entwicklungspolitischen Inlandsarbeit. Eine methodische Bestandsaufnahme von Evaluationen zivilgesellschaftlicher Angebote, 20-21.

Wettstädt, L. \& Asbrand, B. (2014). Handeln in der Weltgesellschaft. Zum Umgang mit Handlungsaufforderungen im Unterricht zu Themen des Lernbereichs Globale Entwicklung. ZEP - Zeitschrift für internationale Bildungsforschung und Entwicklungspädagogik, 37(1), 4-12. doi.org/10.31244/zep.2021.02.08

\title{
Internationales Kolloquium für Promovierende im Kontext von Erwachsenenbildung und Theologie
}

„Wir haben unser Gespräch zwar beendet, aber unsere Debatte ist noch lange nicht abgeschlossen!“ - Dieser Satz eines Teilnehmers fasste die Erfahrungen des zweiten internationalen Kolloquiums für Promovierende im Kontext von Internationaler Erwachsenenbildung und Theologie zusammen. Gastgeber war der Lehrstuhl für Allgemeine Pädagogik der Otto-Friedrich-Universität Bamberg, der das Kolloquium in Zusammenarbeit mit den weiteren Beteiligten organisiert hatte. Am 23. und 24. November 2020 trafen sich 23 Studierende, die sie betreuenden Professorinnen und Professoren sowie Personen, die die Vereinigte Evangelische Mission (VEM) als der Finanzier der drei mit dem Kolloquium in Verbindung stehenden Stipendien. Die Promovierenden aus Indonesien, Kamerun, Kongo/ DRC, Madagaskar, Ruanda, Tansania und Deutschland bearbeiten Promotionsvorhaben bei Prof. Dr. Claudia Jahnel (Ruhr Universität Bochum), Prof. Dr. Annette Scheunpflug (Otto-Friedrich-Universität Bamberg) und Prof. Dr. Wolfgang Jütte (Universität Bielefeld). Themen des Kolloquiums waren: „Transkulturalität, Dekolonisierung und interkulturelle Theologie“ (Jahnel), „Lernen in und von Netzwerken - der Shift von Zentrum und Peripherie zu Knotenpunkten“ (Jütte), und „Globales Lernen Erwachsener in Begegnungen - empirisches Wissen und theoretischer Hintergrund" (Scheunpflug). Die Bildungswissenschaftlerin Dr. Fransina Yoteni aus der GKITP - Evangelical Christian Church in Tanah Papua aus West Papua berichtete aus dem Blickwinkel ihres Kontexts über „Globales Lernen in Situationen von strukturellem Rassismus“.

Den Kern der Studierendengruppe bildete ein dreiköpfiges Team der VEM-Stipendiat/-innen aus Deutschland, Indonesien und Kongo. Sie kommen aus den Fachbereichen Theologie, Kulturwissenschaften und Erziehungswissenschaf- ten und arbeiten in Mitgliedskirchen der VEM an Universitäten oder in Bildungsprogrammen. Ein gemeinsames Doktoratsprogramm - interdisziplinär, international und berufsbegleitend, das ist für die VEM ein Pilotprojekt. Die Promovierenden leben zu Hause in ihren Ländern und treffen sich mindestens einmal jährlich in gemeinsamen Kolloquien - notfalls, so wie jetzt, auch am Bildschirm. Aber auch in der Zwischenzeit sind sie in Kontakt miteinander und stehen in regem Austausch mit ihren Professor/-innen. „Ich bespreche alle Schritte meiner Arbeit mit meinen Kollegen, den anderen Promovenden. Ihre Kommentare sind immer spannend, sie sehen aus ihren Blickwinkeln - sie sind beide Theologen - ja ganz andere Aspekte meines Themas als ich!“, erklärt zum Beispiel Kakule Bulaya, Programmleiter in der CBCA im Kivu mit einem MA in „Educational Quality“. Er untersucht in seiner Doktorarbeit, welche Wirkung die internationalen Begegnungen kirchlicher Mitarbeitenden in der VEM auf ihre Arbeit in seinem Land und auf seine Kirche haben. Alena Höfer, Theologin aus der EKiR, forscht darüber, wie sich das Verständnis von Christologie in transkulturellen Räumen verändert. Und Julius Simaremare, Dozent an der theologischen Hochschule der HKBP, untersucht, in welchen Formen über belastende und traumatische Themen in seinem Kontext kommuniziert wird.

Das nächste Kolloquium ist bereits geplant: Im Herbst 2021 soll es - hoffentlich in physischer Präsenz! - eine „Autumn School“ in Bochum geben. Die Debatten dann im direkten Austausch miteinander weiterführen zu können, darauf freuen sich jetzt schon alle Beteiligten.

Angelika Veddeler \& Andar Parlindungan doi.org/10.31244/zep.2021.02.09 